\title{
Left atrium mass in a patient with breast cancer: a case report
}

\author{
Yu Sugawara ${ }^{1 *}$, Ryuji Okamura ${ }^{2}$ and Shigeki Taniguchi ${ }^{3}$
}

\begin{abstract} warfarin in patients with breast cancer and left atrial thrombus.

Keywords: Apixaban, Thrombus, Left atrial mass

\section{Background}

Left atrial thrombus is one of the differential diagnoses of a left atrial mass and is usually observed in the left atrial appendage. Historically, left atrial thrombi have been treated with heparin and warfarin. Recently, direct oral anticoagulants (DOACs) have been developed, and the superiority of DOACs to warfarin in preventing stroke and reducing bleeding has been demonstrated [1]. However, the efficacy of a DOAC such as apixaban for the treatment of left atrial thrombus has not been established. We experienced a case of a left atrial mass in a patient with breast cancer. The mass was a thrombus that recurred in only 14 days and then resolved completely after approximately 2 months of apixaban administration.
\end{abstract}

Background: Left atrial thrombi have traditionally been treated with heparin and warfarin, and many physicians have limited experience with direct oral anticoagulants such as apixaban. Furthermore, the efficacy of apixaban for the treatment of left atrial thrombi has not been established. We experienced a case of left atrial thrombus formation before breast cancer surgery, which was resolved by apixaban.

Case presentation: Computed tomography for a 74-year-old Japanese woman with breast cancer incidentally revealed a left atrial mass with a root before the breast surgery. The mass was surgically removed and determined to be a thrombus. Before the breast surgery, transthoracic echocardiography was performed again, and the left atrial thrombus had recurred within only 14 days. It resolved after administration of apixaban.

Conclusions: A left atrial thrombus might recur within a very short time. Apixaban might be an alternative to

\section{Case presentation}

A 74-year-old Japanese woman (height $136 \mathrm{~cm}$; weight $40 \mathrm{~kg}$ ) without systemic disease presented to our hospital with a palpable $15-\mathrm{mm}$ mass in her left breast that was identified as stage I breast cancer. Before surgery for

\footnotetext{
* Correspondence: waegurorunsra@hotmail.co.jp

${ }^{1}$ Department of Internal Medicine, Yamatotakada Municipal Hospital, 1-1,

Isono-Cho, Yamatotakada-Shi, Nara-Ken, Japan

Full list of author information is available at the end of the article
}

removal of the mass, a contrast-enhanced pulmonary computed tomography $(\mathrm{CT})$ was performed and showed a mass approximately $30 \times 30 \mathrm{~mm}$ in her left atrium (Fig. 1).

Based on transthoracic echocardiography (TTE), her left ventricle ejection fraction was $65.3 \%$ and her left atrial diameter was $42 \mathrm{~mm}$; the mass was $33 \times 30 \mathrm{~mm}$, it had a root, it was located on the surface of the septal wall, and was oscillating (Fig. 2). An electrocardiogram showed paroxysmal atrial fibrillation. Her chest radiography was normal. Her $\mathrm{CHA}_{2} \mathrm{DS}_{2}$-VASc score was 2, $\mathrm{CHADS}_{2}$ score was 0 , and HAS-BLED score was 1. Laboratory investigations revealed the following: hemoglobin level, $12.8 \mathrm{~g} / \mathrm{dL}$; serum creatinine level, $0.67 \mathrm{mg} / \mathrm{dL}$; estimated glomerular filtration rate, $64.6 \mathrm{~mL} /$ minute $/ 1.73 \mathrm{~m}^{2}$; brain natriuretic peptide, $199.2 \mathrm{pg} / \mathrm{dL}$; international normalized ratio (INR), 1.04; activated partial thromboplastin time (APTT), 32.5 seconds; protein C, $113 \%$; protein S, 75 \%; antinuclear antigen, 40; and lupus anticoagulant, 1.4 seconds. Her levels of cancer antigen 15-3 and NCC-ST 439 were $23.8 \mathrm{U} / \mathrm{mL}$ and $1.3 \mathrm{U} / \mathrm{mL}$, respectively.

Cardiac surgery was performed 16 days after her first visit to remove the left atrial mass. On pathologic examination, the mass consisted of a thrombus, without 


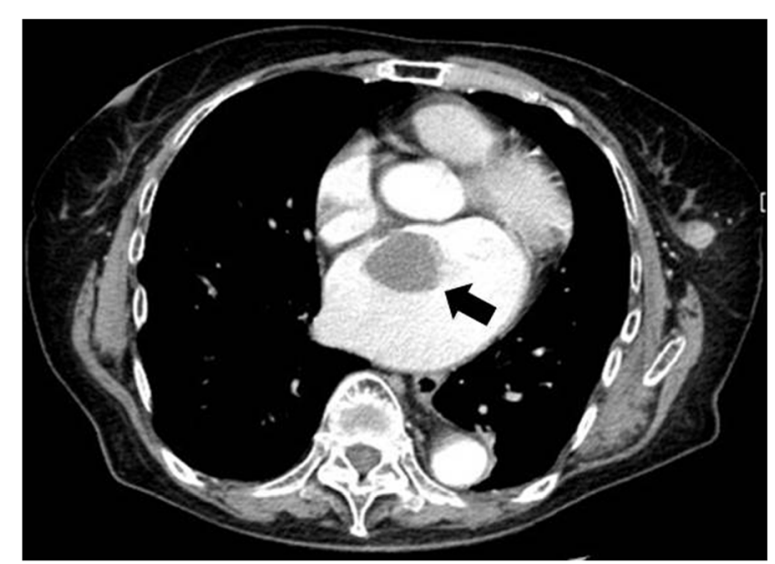

Fig. 1 A $30 \times 30-m m$ mass in the left atrium on contrast-enhanced computed tomography of the chest (black arrow)

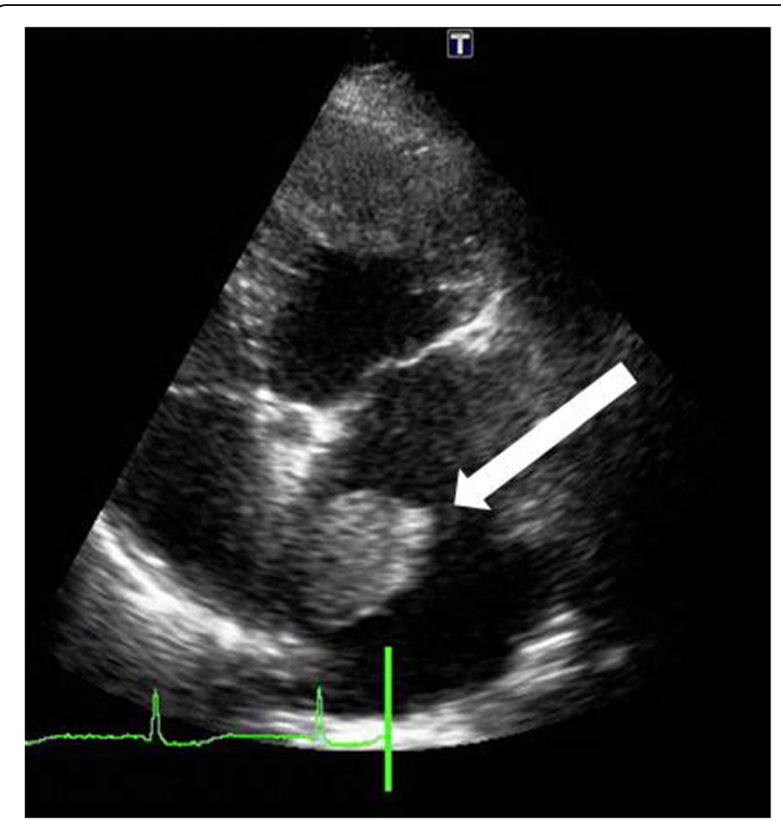

Fig. 2 Transthoracic echocardiography showing a $33 \times 30-\mathrm{mm}$ mass in the left atrium (white arrow)

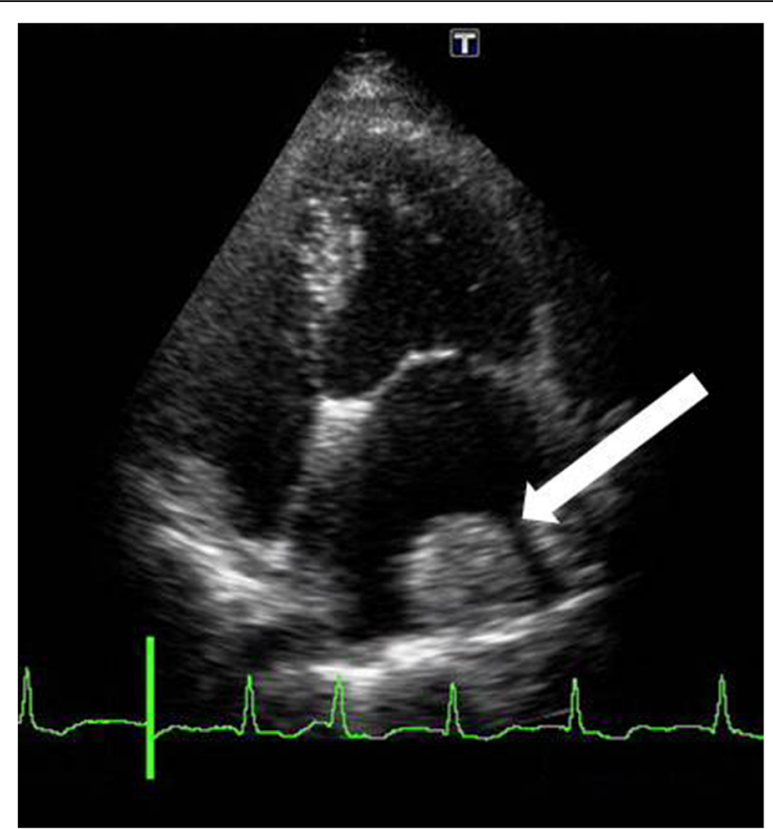

Fig. 4 Fourteen days after cardiac surgery, a thrombus had formed over the lateral left atrium wall (white arrow)

tumor cells (Fig. 3). After removal of the left atrial mass, heparin was initiated, and breast surgery was planned.

Fourteen days after her cardiac surgery, TTE was performed, showing a mass formation in the lateral wall of her left atrium (Fig. 4). The mass was $38 \times 31 \mathrm{~mm}$ and was potentially a thrombus. Therefore, her breast operation was postponed and $1 \mathrm{mg} /$ day of letrozole was administered to prevent progression of the breast cancer. The left atrial thrombus was treated with heparin and warfarin. Because her INR level fluctuated, rivaroxaban was administered. However, owing to gastrointestinal upset, she did not take rivaroxaban; therefore, $5 \mathrm{mg}$ of apixaban twice a day was prescribed. Approximately 2 months later, TTE and contrast-enhanced pulmonary
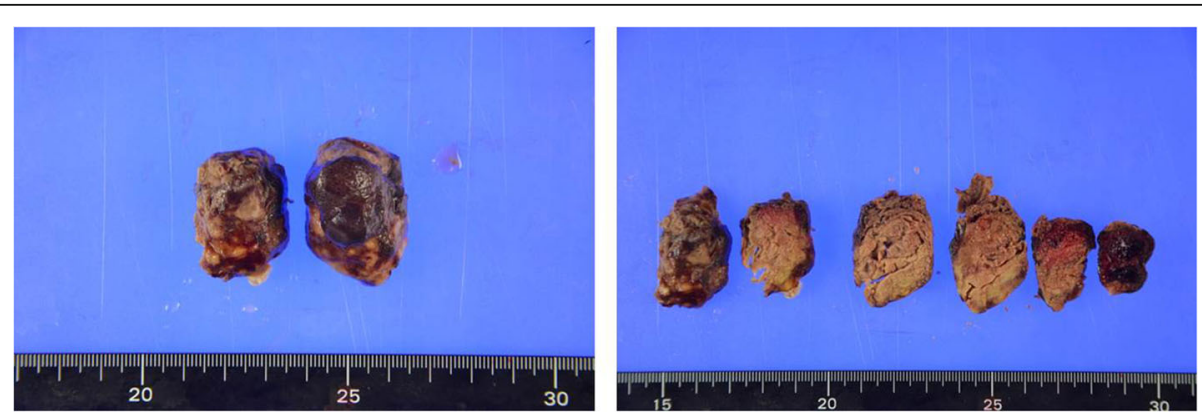

Fig. 3 Left atrial mass pathology showing the mass was composed of a thrombus, without tumor tissue 


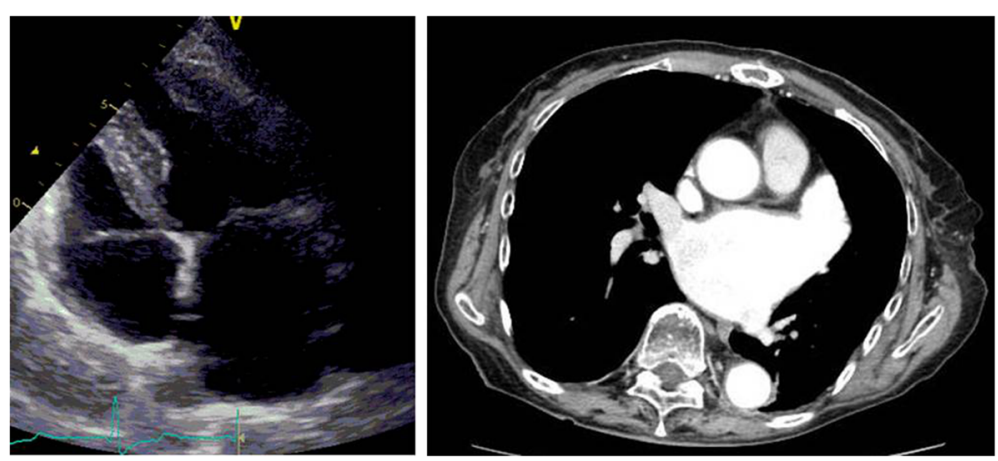

Fig. 5 The cardiac thrombus disappeared on transthoracic echocardiography and contrast-enhanced computed tomography of the chest post-apixaban treatment

CT showed resolution of the left atrial thrombus (Fig. 5). After complete resolution of the left atrial thrombus, her breast surgery was performed. During the anticoagulation therapy, thromboembolism and lethal hemorrhage did not occur.

\section{Discussion}

This case highlighted three important clinical issues. First, apixaban effectively resolved the left atrial thrombus. DOACs such as apixaban have recently been developed and are superior to warfarin for preventing stroke and reducing bleeding [1]. Although there have been some case reports of resolution of a left atrial thrombus with apixaban [2-4], the mechanisms of thrombus resolution are not fully understood. One possible mechanism is that antithrombin is not required for the antithrombotic activity of apixaban, which is a selective factor $\mathrm{Xa}$ inhibitor. It inhibits both free and clot-bound factor Xa as well as prothrombinase activity. It indirectly inhibits platelet aggregation and decreases thrombin generation and fibrin clot development $[5,6]$. In addition, the fibrinolytic system plays a role in thrombus resolution. Because of the limited experience with DOACs for left atrial thrombus resolution, additional cases and further investigation are needed to establish the efficacy of apixaban for this purpose.

Second, the cardiac thrombus relapsed in a very short time after removal. Our patient had paroxysmal atrial fibrillation and cancer. Malignancy has a significant role in a thrombus, and venous and arterial thromboembolisms are common complications for patients with cancer [7]. In a normal coagulation-fibrinolysis system, there is a natural balance between activation and inhibition of procoagulants and anticoagulants. Cancer cells can alter this balance through the production of cancer-related procoagulants such as tissue factor and cancer procoagulant [8]. Tissue factor is a transmembrane glycoprotein and the primer of the physiological coagulation cascade. Cancer procoagulant, a cysteine protease, is a direct activator of factor $\mathrm{X}$ and is found in malignant tissues. Cancer procoagulant, in the presence of factor V, enhances thrombin production [9]. Tumorspecific activation of factor $\mathrm{X}$ might be an important step in the blood coagulation cascade in patients with cancer. In our case, we used a direct Xa inhibitor (apixaban), which resulted in a favorable outcome.

Finally, differential diagnoses of intracardiac mass are a benign tumor, malignant tumor, metastatic tumor, and thrombus. Atrial myxoma is the most common primary intracardiac tumor in adults; two thirds of myxomas arise in the left atrium [10]. However, cardiac thrombi typically occur in the left atrial appendage. In the present case, the left atrial mass had a root and oscillated, which are typical characteristics of myxomas and atypical characteristics of a left atrial thrombus.

\section{Conclusion}

Apixaban is a favorable alternative therapy for patients with a cardiac thrombus.

\section{Abbreviations}

CT: Computed tomography; DOAC: Direct oral anticoagulant; INR: International normalized ratio; TTE: Transthoracic echocardiography

\section{Acknowledgements}

The authors would like to thank Dr Tokiko Nakai (Nara Medical University, Department of Pathology, Kashihara, Japan) who performed the histological examination of the left atrial mass. We would also like to thank Ms Yuki Tsutsumino (Yamatotakada Municipal Hospital) who performed cardiac ultrasonography on multiple occasions. We would like to thank Editage (www.editage.jp) for English language editing.

Funding

This work did not receive any funding.

Availability of data and materials

All data and materials described in this manuscript are fully anonymous.

Authors' contributions

$\mathrm{RO}$ first found the left atrial mass and performed breast surgery. ST performed cardiac surgery. All authors read and approved the final manuscript.

The authors declare that they have no competing interests. 


\section{Consent for publication}

Written informed consent was obtained from the patient for publication of this case report and accompanying images. A copy of the written consent form is available for review by the Editor-in-Chief of this journal.

\section{Ethics approval and consent to participate}

This study has been performed in accordance with the Declaration of Helsinki and has been approved by ethics committee of Yamatotakada Municipal Hospital. The committee's reference number is H28-4.

\section{Author details}

'Department of Internal Medicine, Yamatotakada Municipal Hospital, 1-1, Isono-Cho, Yamatotakada-Shi, Nara-Ken, Japan. ' Department of Surgery, Yamatotakada Municipal Hospital, 1-1, Isono-Cho, Yamatotakada-Shi, Nara-Ken, Japan. ${ }^{3}$ Department of Cardiovascular Surgery, Nara Medical University, 840, Shijo-Cho, Kashihara-Shi, Nara-Ken, Japan.

Received: 25 January 2016 Accepted: 29 September 2016

\section{Published online: 19 October 2016}

\section{References}

1. Granger CB, Alexander JH, McMurray JJ, Lopes RD, Hylek EM, Hanna M, et al. Apixaban versus warfarin in patients with atrial fibrillation. N Engl J Med. 2011:365:981-92. doi:10.1056/NEJMoa1107039.

2. Kawakami T, Kobayakawa $H$, Ohno H, Tanaka N, Ishihara H. Resolution of left atrial appendage thrombus with apixaban. Thromb J. 2013;11:26. doi:10.1186/1477-9560-11-26

3. Dobashi S, Fujino T, Ikeda T. Use of apixaban for an elderly patient with left atrial thrombus. BMJ Case Rep. 2014;2014. doi: 10.1136/bcr-2014-203870.

4. Miwa Y, Minamishima T, Sato T, Sakata K, Yoshino H, Soejima K. Resolution of a warfarin and dabigatran-resistant left atrial appendage thrombus with apixaban. J Arrhythm. 2016;32:233-5. doi:10.1016/j.joa.2016.01.009.

5. Mandernach MW, Beyth RJ, Rajasekhar A. Apixaban for the prophylaxis and treatment of deep vein thrombosis and pulmonary embolism: an evidencebased review. Ther Clin Risk Manag. 2015;11:1273-82. doi:10.2147/TCRM. S68010

6. Mousa SA. Role of current and emerging antithrombotics in thrombosis and cancer. Drugs Today (Barc). 2006:42:331-50.

7. Falanga A. Thrombophilia in cancer. Semin Thromb Hemost. 2005:31:104-10.

8. De Cicco M. The prothrombotic state in cancer: pathogenic mechanisms. Crit Rev Oncol Hematol. 2004;50:187-96.

9. Kyriazi V. Breast cancer as an acquired thrombophilic state. J Breast Cancer 2012;15:148-56. doi:10.4048/jbc.2012.15.2.148.

10. Lee WC, Huang MP, Fu M. Multiple intracardiac masses: myxoma, thrombus or metastasis: a case report. J Med Case Rep. 2015;9:179. doi:10.1186/ s13256-015-0650-4.

\section{Submit your next manuscript to BioMed Central and we will help you at every step:}

- We accept pre-submission inquiries

- Our selector tool helps you to find the most relevant journal

- We provide round the clock customer support

- Convenient online submission

- Thorough peer review

- Inclusion in PubMed and all major indexing services

- Maximum visibility for your research

Submit your manuscript at www.biomedcentral.com/submit 